\title{
Las tics como herramientas para la prevención de las enfermedades digestivas en los estudiantes de la I.E.D San José de Pueblo Viejo bajo el modelo de la investigación como estrategia pedagógica (IEP) 1
}

\section{The tics as tools for the prevention of digestive diseases in the students of the I.E.D San Jose de Pueblo Viejo under the model of research as a pedagogical strategy (IEP)}

DOI: http://dx.doi.org/10.17981/cultedusoc.9.3.2018.77

\author{
Artículo de investigación. Fecha de recepción: 15/06/2018. Fecha de aceptación: 27/11/2018 \\ Adela Moreno'; \\ Gloria Juvinao y Yeison Jarma ${ }^{3}$ \\ Institución Educativa Departamental San José de Pueblo Viejo (Colombia)
}

Para citar este artículo:

Moreno, A., Juvinao, G. y Jarma, Y. (2018). Las tics como herramientas para la prevención de las enfermedades digestivas en los estudiantes de la I.E.D san José de pueblo viejo bajo el modelo de la investigación como estrategia pedagógica (IEP). Cultura. Educación y Sociedad 9(3), 655-660. DOI: http://dx.doi.org/10.17981/cultedusoc.9.3.2018.77

Resumen

El desarrollo de los buenos hábitos alimenticios en los estudiantes es importante para disminuir algunas enfermedades del sistema digestivo y conseguir una alimentación balanceada en los estudiantes de la básica primaria. Este estudio tiene como objetivo sensibilizar acerca de la importancia de la adecuada alimentación en los estudiantes y su relación directa con las enfermedades asociadas al sistema digestivo. Se realizó un abordaje bajo el enfoque cualitativo utilizando el tipo de investigación -acción participativa, desarrollando el modelo de la investigación como estrategia pedagógica IEP, desde un diseño descriptivo, donde se utilizaron técnicas como observación participante y herramientas como diario de campo y entrevistas. Se realizaron encuestas a los estudiantes y padres de familia.

Esta muestra fue tomada en 60 estudiantes de La I.E.D San José de Pueblo Viejo del grado quinto de la sede $\mathrm{N}^{\circ} 2$ y en 60 padres de familias. Se obtuvo como resultado una vez realizadas las visitas y la sensibilización, que tanto padres y estudiantes pudieron tomar medidas correctivas para la disminución de las enfermedades digestivas, mediante hábitos tales como: lavarse las manos, en los posibles alimentarse en las mismas horas y a tiempo, lavar muy bien los alimentos, no cortar las cadenas de frio entre otras.

Palabras clave: hábitos alimenticios, sistema digestivo, enfermedades digestivas

\section{Abstract}

The development of good eating habits in students is important to alleviate some diseases of the digestive system and obtain a balanced diet in elementary school students. The objective of this study is to raise awareness about the relationship of the food source in students and its direct relationship with diseases associated with the digestive system. An approach was made under the qualitative approach using the type of research -a participatory action, the development of the research model as an IEP pedagogical strategy, from a descriptive design, where techniques such as observation and tools such as field diary and interviews are used. He associated with students and parents.

It is taken into account in 60 students of La I.E.D. San José de Pueblo Viejo of the fifth grade of the N $\circ 2$ headquarters and 60 parents of families. They were found as a result of visits and sensitization, which both parents and students took corrective measures for the reduction of digestive diseases, while the habits of the stories such as: washing hands, in possible feeding in the same hours and time, wash the food very well, not cut the cold chains among others.

Keywords: dietary habits, digestive system, digestive diseases

1 Este artículo ha sido derivado del Programa de Fortalecimiento de la Cultura Ciudadana y Democrática CT+I a través de la IEP apoyada en TIC en el Departamento de Magdalena: CICLON

2 Líder del grupo de investigación "Guardianes de la Salud".

3 Docentes de la Institución Educativa Departamental San José de Pueblo Viejo y del grupo de investigación "Guardianes de la Salud".

- The author; licensee Universidad de la Costa - CUC.

Cultura, Educación y Sociedad vol. 9 no. 3, pp. 655-660. Diciembre, 2018

Barranquilla. ISSN 2389-7724 Online 


\section{Introducción}

Al tener en cuenta la niñez como una de las primeras etapas del ser humano, se puede resaltar que es importante ofrecer una alimentación saludable en esta etapa, contribuyendo de manera importante al adecuado crecimiento y desarrollo de los niños. Diversos estudios han observado que un excesivo consumo de alimentos de alta densidad energética, ricos en grasas saturadas, azúcar y sal, y la falta de actividad física, representan en la actualidad el estilo de vida de gran parte de los ninos. Este desequilibrio entre la ingesta y el gasto energético, sostenido por períodos prolongados de tiempo, ha sido reconocido internacionalmente como la principal causa de la elevada y creciente prevalencia de obesidad (Olivares, Bustos, Moreno, Lera, \& Cortez, 2006). La obesidad en la infancia es considerada por algunos autores como una epidemia global que puede acarrear graves consecuencias para la salud pública, teniendo en cuenta que la tendencia al sobrepeso en los niños se refleja en los adultos (Carrillo, Pita, Díaz, Mercader y Wong, 2009).

Las desviaciones en el crecimiento temprano, ya sea por el bajo peso y el retardo del crecimiento o por sobrepeso y obesidad, pueden minar la salud de los niños y, por ende, su bienestar (Black, Creed, 2012).

La adopción de hábitos alimenticios saludables en los niños ayuda a prevenir el sobrepeso y la obesidad; la desnutrición crónica, el retardo del crecimiento, la anemia, entre otros (Santos, 2005).

En la sociedad los padres de familia tienen varios compromisos con sus hijos, uno de ellos es brindar buenos hábitos de alimentación. Estos hábitos que se consideran buenos, deben presentarse desde el inicio de la alimentación de los niños, debido a que será el patrón de alimentación que estos tendrán a lo largo de su vida. Es por ello que nace el interés de educar de manera correcta desde el inicio de esta necesidad vital.
Burgos (2007) planteaba que en la mayoría de la población infantil que se encontraba en edad escolar era claro observar una continuación de malos hábitos alimenticios: como la comida chatarra y procesada, dejando a un lado la comida casera y los alimentos orgánicos parecen estar por fuera del menú de las mesas. Al parecer, las nuevas generaciones no están al tanto de estas opciones alimenticias, que podrían ayudarles a lograr un mejor desempeño, tanto en la escuela como en su vida diaria.

Así como se evalúa el crecimiento lineal a través de los años, debe haber un seguimiento en el peso de los niños. El peso adecuado se establece con base en la estatura, ya que no existe un peso ideal para la edad (Ruiz-Martínez, Álvarez-Martínez y RuizJaramillo, 2012).

El tener malos hábitos a nivel de la alimentación puede desprender ciertas alteraciones en la formación y crecimiento del niño, además está fuertemente relacionado con un desequilibrio en el funcionamiento normal del organismo.

\section{Enfermedades del sistema digestivo}

$\mathrm{Al}$ ingerir los alimentos, estos se descomponen de tal manera para producir y nutrir células y suministrar energía donde lo solicite el cuerpo. A este proceso se le denomina digestión.

El sistema digestivo es un conjunto de órganos huecos que se encuentran unidos en un tubo largo y retorcido. Este comienza en la boca y finaliza en el ano, también incluye: el esófago, el estómago, los intestinos delgado y grueso. El hígado, la vesícula y el páncreas también participan, estos tres órganos producen jugos que ayudan en el proceso de la digestión (Capuz, 2017).

$\mathrm{El}$ aparato digestivo, el medio ambiente y la mayoría de las enfermedades como los virus y bacterias, etc. que se logran desarrollar en él, se encuentran relacionadas. Evitarlas depende mucho del cuidado que cada uno tenga con su aparato digestivo (Cabello, 2007). 
La salud está influida por variedad de factores, entre los cuales cabe destacar la alimentación, que incide de manera especial en el desarrollo armónico infantil. El consumo ha de ser suficiente, natural y equilibrado, ya que la calidad de vida dependerá en gran medida de los nutrientes que se aporten al organismo mediante la alimentación, la cual permite el crecimiento y la realización de las funciones biológicas y psicológicas (Romero, 2013).

Una enfermedad del sistema digestivo es considerada cualquier contrariedad en la salud que intervenga los procesos del aparato digestivo. Los síntomas pueden ir de leves a graves. En algunos casos pueden desarrollar cáncer, intolerancia a los lácteos, síndrome de intestino irritable, colecistitis y colangitis, cálculos biliares, reflujo gastroesofágico (ERGE), entre otros problemas digestivos (National Library of Medicine, 2017).

Es clave que los niños tengan una nutrición acorde con su edad, esta traerá consigo muchos beneficios, contribuirá en su crecimiento y desarrollo, en los casos de malnutrición; exceso o defecto, se debe acudir a un médico a tiempo, debido a que, si no se tiene un rápido control, con el tiempo podría desencadenar enfermedades asociadas, como hipertensión arterial, patologías coronarias y/o renales y osteoporosis (Herrera, 2014).

\section{¿Por qué es importante la digestión?}

Para tener una buena salud, es importante tener una buena digestión, lo cual afectará de manera positiva el peso y un metabolismo activo. Esto se debe a que cuando se realiza una buena digestión obtenemos los micronutrientes, vitaminas, minerales y calorías indispensables para el organismo. Si la digestión no es buena, empiezan a aparecer toxinas las cuales producen enfermedades o problemas de salud (Guzmán, 2007; Castillo, 2012)

Una buena dieta es importantísima para mantener tu salud en estado óptimo, pero no lo es menos el papel que desempeña el agua dentro de la digestión.
El sistema digestivo es la estrella principal del proceso de la digestión, que consiste en la transformación de los alimentos previamente ingeridos, en sustancias más sencillas para ser absorbidos (Lozada, 2011).

En este caso, el agua, puede ser la mejor herramienta para favorecer el proceso ya que, entre otras funciones, se encarga de diluir los nutrientes de los alimentos, y transportarlos a las células, así como los productos de desecho desde estas.

Gracias a la digestión el cuerpo pueda usar los alimentos como fuente de energía, y para formar y alimentar las células.

\section{¿Cómo se digieren los alimentos?}

Los órganos que hacen parte del tracto digestivo de manera ordenada son la boca, el esófago, el estómago, el intestino delgado, el intestino grueso; También denominado como colon, finalizando con el recto y el ano. Estos órganos huecos (la boca, el estómago y el intestino delgado) en su interior están revestidos por una membrana llamada mucosa. La mucosa contiene glándulas microscópicas que producen jugos que favorecen la digestión de los alimentos que se consumen a diario. Otro de los aspectos del tracto digestivo es la capa muscular suave que ayuda a transformar los alimentos y conducirlos a lo largo del tubo (National institute of diabetes and digestive and kidney diseases NIDDK, 2008).

Los procesos digestivos, tanto mecánicos como químicos, empiezan en la boca, y se van continuando ordenadamente en el trayecto hacia el ano, transformando paulatinamente los alimentos ingeridos en una masa homogénea formada por nutrientes asimilables (Hernández-Gil y FernándezTresguerres, 2010).

La digestión es comprendida como la mezcla de los alimentos, su travesía a través del tracto digestivo y la descomposición química de las moléculas grande a pequeñas. Todo esto comienza en la boca, al masticar e ingerir los alimentos. Terminando en el intestino delgado (NIDDK, 2008). 


\section{Metodología}

Se realizó un estudio de tipo cualitativo, mediante la investigación -acción participativa, desde un diseño descriptivo. La investigación cualitativa busca conocer e interpretar la realidad de los participantes a través de sus propias experiencias, entregando una información subjetiva del fenómeno de estudio. (Hernández, Fernández, y Baptista, 2010). Por su parte el modelo de investigación como estrategia pedagógica plantea una producción de conocimiento basado en la reflexión de los participantes, teniendo en cuenta la participación activa de maestros en el proceso de enseñanza y acompañamiento tecnológico, guiando al estudiante en su proceso de investigación. Generando nuevo saber y conocimiento sobre una realidad determinada en un proceso de aprendizaje colaborativo (Mejía, 2011).

TABLA 1

Distribución de la población

\begin{tabular}{cc}
\hline Genero & Número de estudiantes \\
\hline Masculino & 22 \\
Femenino & 28 \\
TOTAL & $\mathbf{5 0}$ \\
\hline
\end{tabular}

Fuente: elaboración propia.

Es importante mencionar que los estudios con un diseño descriptivo están encaminados a resaltar las características de la población sujeto de estudio o los fenómenos que sean sometidos al análisis. Bajo un estudio de tipo descriptivo, debido a que no se han realizado estudios previos en la institución. (Hernández, Fernández, y Baptista, 2010). La población tomada para el estudio, fue una muestra representativa de la comunidad estudiantil, de cincuenta (50) estudiantes de La I.E.D San José de Pueblo Viejo del grado quinto de la sede $n^{\circ} 2$, distribuidos entre hombres y mujeres; se realizó un muestreo de tipo intencional, escogiendo los estudiantes que presentaban características relacio- nadas con el objeto de estudio, para observar la distribución de la muestra (tabla 1).

A la hora de recoger los datos se utilizaron la observación participante como técnica y las entrevistas y diario de campo como herramientas de recolección cualitativas que implican que el docente tome un rol activo en el proceso de investigación, registrando cada elemento observado en el diario de campo, con relación a la variable de estudio.

La metodología del proyecto de investigación se realizó a partir de los recorridos de las trayectorias de indagación, basados en la integración de la IEP apoyada en TIC al aula, donde se establecieron cinco (5) trayectos que especifican la ejecución como la conformación de los grupos de aprendizaje, seguidamente de la formulación de la pregunta problema, lo que conlleva a la discusión del grupo de aprendizaje colaborativo, dando inicio al aprendizaje situado. Luego el grupo plantea el problema específico e identifica las causas y los efectos en cuanto la teoría que será investigada con las herramientas TIC; tabletas como elementos didácticos y las metas trazadas para darle solución a la pregunta problema planteado, como el diseño de los recorridos que permiten iniciar la indagación por parte del grupo de aprendizaje colaborativo, escogiendo las diversas técnicas y herramientas mencionadas anteriormente, finalmente se da espacio para la recolección de información a través de la negociación culturas, el contraste y la organización de los conocimientos frente a la teoría (Mejía, 2011).

\section{Resultados}

A continuación, se presentan los hallazgos del estudio, a partir de la trayectoria recorrida bajo el modelo de la IEP, se evidencio que los estudiantes de la IED San José de Pueblo Viejo $\mathrm{N}^{\circ} 2$, presentan dificultades especificas del sistema digestivo y observando deficiencias en los hábitos alimenticios y de higiene, lo cual es determinante para prevenir este tipo de afecciones. 
Al identificar las problemáticas presentadas por la comunidad estudiantil de la institución los docentes realizaron un proceso de sistematización donde reflexionaron algunos de los hábitos observados en los estudiantes y en las entrevistas y visitas realizadas a los padres de familia sobre los hábitos de higiene y alimenticios, donde se pudo identificar la necesidad de generar espacios de formación, sensibilización y concientización sobre los buenos habito. Una vez ejecutado el proyecto y a partir de los conocimientos adquiridos por el proyecto ciclón en materia de investigación y TIC, se reunieron para diseñar estrategias transversales a las diferentes áreas del currículo que pudieran ayudar a esta sensibilización.

En ese sentido y mediante el refuerzo desde las diversas asignaturas se logró tener un impacto positivo. En cuanto a las estrategias se integró la IEP, donde los docentes a partir de la temática orientadora de la asignatura realizaban espacios de discusión para generar preguntas relacionadas con el tema por parte de los estudiantes, partiendo de las preguntas de sentido común, se orientaba a identificar el problema y se diseñaban las trayectorias de indagación, donde se utilizaban diferentes herramientas para recoger la información, desde consultas en internet por medio de las tabletas como herramientas didácticas, hasta pequeñas entrevistas realizadas a la comunidad.

La información recolectada dejó en evidencia que, al integrar la teoría con la práctica, el estudiante interioriza los conocimientos, se apropia de ellos y de esta manera le es más fácil socializar las experiencias, utilizando recursos didácticos de sitios web, aplicaciones móviles, las tabletas, procesadores de texto, blogs, y canales de YouTube, entre otros recursos.

Se realizó un proceso de propagación y divulgación de la información, donde el grupo compartió con la comunidad educativa por medio de ferias, devolviendo el conocimiento hallado a la comunidad asimismo, se socializo a la comunidad y padres de familia, sobre los hallazgos en el proceso.

Finalmente se logró una variación en los cambios de hábitos los cuales paulatinamente se verán reflejados en la prevención de algunas enfermedades.

\section{Discusión}

La falta de una buena alimentación por parte de los estudiantes del IED San José de Pueblo Viejo, está relacionada directamente con las alteraciones y el adecuado funcionamiento del sistema digestivo, es muy preocupante ver como niños de tan corta edad empiezan a presentar problemas de salud por falta de una alimentación adecuada. Maestros y directivos se han puesto en marcha respecto al tema por medio de la educación e investigación, desarrollando investigaciones bajo el modelo de la investigación como estrategia pedagógica IEP, buscando enlazar los conocimientos establecidos académicamente y la información que logra otorgar la comunidad desde su percepción y las investigaciones relacionadas (Mejía, 2011), con la finalidad de lograr una mejora en la salud de los estudiantes e incentivar los hábitos investigativos en los distintos grupos de aprendizaje. Se espera que dentro de unos años los problemas por falta de alimentación entre los estudiantes de la institución hayan disminuido hasta un $70 \%$. Integrando conocimientos y distintas acciones, así como la metodología de la IEP donde no sólo se dé a conocer sobre los problemas escogido o relevantes, si no, se logren ampliar las ideas, objeciones y respuestas (Mejía, 2011) puntualmente sobre la alimentación adecuada. Por lo tanto no se puede desconocer a los estudiantes desde el aspecto social, asimismo, a la comunidad como actores en una mesa de trabajo, donde contribuyen a la búsqueda de proyectos que contribuyen al mejoramiento de problemáticas. 


\section{Referencias}

Black, M. y Creed-Kanashiro, H. (2012). ¿Cómo alimentar a los niños?: La práctica de conductas alimentarias saludables desde la infancia. Revista Peruana de medicina experimental y salud pública, 29(3), 373-378.

Burgos, N. (2007). Alimentación y nutrición en edad escolar. Revista Digital Universitaria, 8(4), 1067-6079.

Cabello, R. (2007). Microbiología y parasitología humana/Microbiology and $\mathrm{Hu}$ man Parasitology: Bases etiologicas de las enfermedades infecciosas y parasitarias/Etiological Basis of Infectious and Parasitic Diseases. Argentina: Médica Panamericana.

Castillo Reinosa, M. (2012). La nutrición en el marco de la educación para la salud, un instrumento para incidir en el aumento de resiliencia y en el proyecto de vida del educando. [Doctoral dissertation]. Universidad Nacional.

Capuz, J. (2017) Estrategias educativas para la prevención de la enfermedad diarreica aguda en los niños menores de cinco años que asisten al centro de salud morete puyo. [Tesis de pregrado]. Universidad Regional Autónoma de los Andes.

Carrillo S., M., Pita R., G., Díaz, M. E., Mercader, O. y Wong, I. (2009). Evaluación nutricional de niños de 10 a 14 meses de edad. Revista Cubana de Pediatría, 81(3). 1-10.

Losada, I. (2011). Nutrición para el bienestar. Morrisville: Lulu.com.

Guzmán, R. (2007) Nutrición y rendimiento físico en bailarines profesionales. [Tesis de pregrado]. Universidad de Chile.

Hernández, R., Fernández, C. y Baptista, P. (2010). Metodología de la investigación. México, D.F.: Mac GarwHill.

Hernández-Gil, I. y Fernández-Tresguerres, I. (2010). FIntroducción al aparato digestivo. Cavidad bucal. HUMANA, 4. 682.
Herrera, N. (2014). Estudio comparativo del estado nutricional con el desarrollo mótriz de niñas y niños de 3 a 5 años del Centro de Educación Inicial Muñequitos de Lumbisi. [Bachelor's thesis]. Universidad de las Fuerzas Armadas ESPE.

Mejia, R. (2011). La investigación como estrategia pedagógica una apuesta por construir pedagogías críticas en el siglo XXI. Praxis \& Saber, 2(4). 127-177

National Institute of Diabetes and Digestive and Kidney Diseases [NIDDK] (2008). Aparato digestivo y su funcionamiento. (08-2681S). Recuperado de https://www. niddk.nih.gov/health-information/informacion-de-la-salud/enfermedades-digestivas/aparato-digestivo-funcionamiento

National Library of Medicine (2017) Enfermedades del sistema digestivo. MedlinePlus. Recuperado de https://medlineplus. gov/spanish/digestivediseases. html\#cat_51

Noguera, D., Márquez, J. C., Campos, I. y Santiago, R. (2013). Alimentación complementaria en niños sanos de 6 a 24 meses. Archivos venezolanos de puericultura y pediatría, 76(3), 126-135.

Olivares, S., Bustos, N., Moreno, X., Lera, L. y Cortez, S. (2006). Actitudes y prácticas sobre alimentación y actividad física en niños obesos y sus madres en Santiago, Chile. Revista chilena de nutrición, 33(2), 170-179.

Romero, R., (2013) Promoción de hábitos alimentarios saludables de la escuela. [Tesis de pregrado]. Universidad de Valladolid.

Ruiz-Martínez, E., Álvarez-Martínez, I. y Ruiz-Jaramillo, M. (2012). Hábitos de alimentación en niños con sobrepeso y obesidad. Pediatría de México, 14(3), 124132 .

Santos, S. (2005). La Educación Física escolar ante el problema de la obesidad y el sobrepeso. Revista Internacional de Medicina y Ciencias de la Actividad Física $y$ del Deporte, 5(19). 179-199. 